\title{
A single server queueing model with self-generation of priorities, customer induced interruption and retrial of customers
}

\author{
${ }^{1}$ Jomy Punalal, ${ }^{2}$ S. Babu \\ ${ }^{1}$ Assistant Professor, ${ }^{2}$ Associate Professor \\ ${ }^{1,2}$ Department of Mathematics, \\ ${ }^{1,2}$ University College, Thiruvananthapuram, Kerala, India \\ Email: jomypunalal@gmail.com ${ }^{1}$,drbabu.s@gmail.com ${ }^{2}$
}

\begin{abstract}
In this article we consider a single server queueing model in which customers arrive according to Poisson process. At the time of arrival, all customers are classified as ordinary. If the server is busy the arriving customers enter an orbit of infinite capacity. Each customer in the orbit tries, independently of each other, to access the server at a constant rate $\sigma$. Each customer in the orbit, independently of others, generate priority with inter-occurrence time exponentially distributed with parameter $\gamma$. A priority generated customer is immediately taken for service if the server is free. Else, such a customer is placed in a waiting space $A_{1}$ of capacity 1 which is reserved only for priority generated customers. We consider a customer induced interruption while service is going on. The interruption occurs according to a Poisson process. The interrupted customers will enter into a buffer $B_{1}$ of finite capacity $K$ and they will spend a random period of time for completion of interruption. The duration of the interruption of customers in $B_{1}$ follows an exponential distribution. The service facility consists of one server and the duration of service times of ordinary, priority and interruption completed customers follows an exponential distribution with appropriate representations. Various performance measures are obtained for an appropriate system designing.
\end{abstract}

Index Terms: Customer induced interruption; Level Dependant Quasi-Birth-Death Process; Matrix Analytic Method; Retrial Queues; Self-generation of priorities.

\section{INTRODUCTION}

Queueing models with self-generation of priorities, customer induced interruption and retrial of customers has wide applications. Some applications in health care systems are mentioned in articles [3], [16], [4],[11] and in books [14], [7], [15]. The pioneer works on selfgeneration of priorities are [5],[9],[8]. There is a survey on queues with interruption [10] and customer induced interruption appeared in [6]. Retrial queues are extensively investigated in [18], [2], [1]. The present paper discusses a single server queueing system in which the arrival of customers form a Poisson process and service times are exponentially distributed. Using the matrix-analytic method (refer [12] for details) we study this model and some measures of system performance in the steady state are derived and analyzed graphically.

\section{MODEL DESCRIPTION}

We consider a single server queueing system in which customers arrive according to Poisson process with parameter $\lambda$. An arriving customer enters service immediately if the server is free and if the server is busy, the customer enters an orbit of infinite capacity. Each customer in the orbit independently tries to access the server according to Poisson process with parameter $\sigma$. A retrial customer who finds the server busy returns to the orbit with probability $\delta(<1)$ and leaves the system forever with probability $1-\delta$. The customers in the orbit can generate priority according to Poisson process with parameter $\gamma$. A priority generated customer is immediately taken for service if the server is free, otherwise, move to a waiting space $A_{1}$ of capacity one which is reserved only for priority generated customers. If the waiting space $A_{1}$ is occupied with a previous priority generated customer, the new priority generated customer will leave the system forever. We also consider a customer induced interruption when service is going on and the interruption occurs according to a Poisson process with parameter $\theta$. The interrupted customers will enter into a buffer $B_{1}$ of finite capacity $K$ if there is space available and the customer will be lost forever if no space for the customer in buffer $B_{1}$. The service provided here is non-preemptive and the service times are exponentially distributed with parameters $\mu_{1}, \mu_{2}$ and $\mu_{3}$ for ordinary, priority generated and interruption completed customers respectively. When an interruption occurs, the customer currently in service will be forced to leave the service facility and the freed server is ready to offer services to other customers. The interrupted customer will spend a random period of time for completion of interruption and it follows an exponential distribution with parameter $\eta$ and customers who completed the interruption will move to a buffer $B_{2}$ whose size is also $K$. We assume that priority generated customers will never undergo interruption and not more than one interruption is allowed for a customer during service. 


\section{Available online at www.ijrat.org}

Also assume that the sum of a number of customers in $B_{1}$ and $B_{2}$ should be less than or equal to $K$. Otherwise, if buffer $B_{2}$ is full, we cannot accommodate one more interruption-completed customer from the buffer $B_{1}$.

The model is studied as a Quasi Birth-Death (QBD) process and Matrix Geometric solution is obtained. The following notations are used to describe the model:

$N_{1}(t)$ - Number of customers in the orbit at time $t$,

$N_{2}(t)$ - Number of busy servers at time $t$.

$S(t)-\left\{\begin{array}{lr}1, & \text { server busy with ordinary customers } \\ 2, & \text { server busy with priority generated customers } \\ 3, & \text { server busy with interruption completed customers }\end{array}\right.$

$N_{3}(t)$ - Number of priority generated customers waiting for service at time $t$.

$N_{4}(t)$ - Number of interruption completed customer in buffer $B_{2}$ at time $t$.

$N_{5}(t)$ - Number of interrupted customers in buffer $B_{1}$ at time $t$.

Under the assumptions on arrival and service processes $\{\chi(t): t \geq 0\}$ where

$\chi(t)=\left\{N_{1}(t), N_{2}(t), S(t), N_{3}(t), N_{4}(t), N_{5}(t)\right\}$ form a continuous time Markov chain on the state space $\Omega=L_{1}(i) \cup L_{2}(i)$, where $L_{1}(i)=\left\{\left(i, 0, w, b_{2}, b_{1}\right)\right.$ : $\left.i \geq 0 ; w=0,1 ; b_{2}, b_{1}=0,1,2, \cdots, K ; b_{2}+b_{1} \leq K\right\}$, $L_{2}(i)=\left\{\left(i, 1, s, w, b_{2}, b_{1}\right): i \geq 0, s=1,2,3 ; w=\right.$ 0,$\left.1 ; b_{2}, b_{1}=0,1,2, \cdots, K ; b_{2}+b_{1} \leq K\right\}$.

By partitioning the state space into levels with respect to the number of customers in the orbit, the generator of above Markov process is of the form:

$Q=$

$=\left[\begin{array}{llllllll}A_{10} & A_{0} & \cdot & \cdot & \cdot & \cdot & \cdot & \cdot \\ A_{21} & A_{11} & A_{0} & \cdot & \cdot & \cdot & \cdot & \cdot \\ & A_{22} & A_{12} & A_{0} & \cdot & \cdot & \cdot & \cdot \\ & & \ddots & \ddots & \ddots & & & \\ \cdot & \cdot & \cdot & A_{2 N} & A_{1 N} & A_{0} & \cdot & \cdot \\ \cdot & \cdot & \cdot & \cdot & A_{2 N+1} & A_{1 N+1} & A_{0} & \cdot \\ . & \cdot & \cdot & \cdot & \cdot & \ddots & \ddots & \ddots\end{array}\right]$

where $\dot{A_{0}}, A_{10}, A_{2 i}, \dot{A_{1 i}}, \quad \dot{i}=1,2,3, \cdots \quad$ are square matrices of order $4(K+1)(K+2)$ and defined as follows:

\section{Matrix $A_{10}$ has the following transitions:}

Entries of $A_{10}$ are the transitions within level 0.

Let $s=1,2,3 ; w=0,1 ; b_{2}, b_{1}=1,2,3, \ldots K$;

$b_{2}+b_{1} \leq K$.

- $\left(0,0, w, b_{2}, b_{1}\right) \stackrel{-\lambda-b_{1} \eta}{\longrightarrow}\left(0,0, w, b_{2}, b_{1}\right) \quad$; if $b_{1}=K, b_{2}+b_{1}=K, b_{2}+b_{1}<K$

- $\left(0,0, w, b_{2}, b_{1}\right) \stackrel{-\lambda}{\longrightarrow}\left(0,0, w, b_{2}, b_{1}\right) \quad$; otherwise

- $\left(0,1, s, w, b_{2}, b_{1}\right) \stackrel{-\lambda-b_{1} \eta}{\longrightarrow}\left(0,1, s, w, b_{2}, b_{1}\right)$; if $b_{1}=K, b_{2}+b_{1}=K, b_{2}+b_{1}<K$
- $\left(0,1, s, w, b_{2}, b_{1}\right) \stackrel{-\lambda}{\longrightarrow}\left(0,1, s, w, b_{2}, b_{1}\right)$; otherwise

\section{Matrix $A_{0}$ has the following transitions:}

Entries of $A_{0}$ are the transitions from $i$ to $(i+1)$. Let $s=1,2,3 ; w=0,1 ; b_{2}, b_{1}=1,2,3, \ldots K ; b_{2}+$ $b_{1} \leq K$.

- $\left(i, 0, w, b_{2}, b_{1}\right) \stackrel{\lambda}{\longrightarrow}\left(i+1,0, w, b_{2}, b_{1}\right)$;

- $\left(i, 1, s, w, b_{2}, b_{1}\right) \stackrel{\lambda}{\longrightarrow}\left(i+1,1, s, w, b_{2}, b_{1}\right)$;

\section{Matrix $A_{2 i}$ has the following transitions:}

Entries of $A_{2 i}$ are the transitions from $i$ to $(i-1)$. Let $s=1,2,3 ; b_{2}, b_{1}=1,2,3, \ldots K ; b_{2}+b_{1} \leq K$.

- $\quad\left(i, 0,0, b_{2}, b_{1}\right) \stackrel{i \gamma}{\longrightarrow}\left(i-1,0,1, b_{2}, b_{1}\right)$;

- $\quad\left(i, 1, s, 0, b_{2}, b_{1}\right) \stackrel{i \gamma}{\longrightarrow}\left(i-1,1, s, 1, b_{2}, b_{1}\right)$;

- $\quad\left(i, 0,1, b_{2}, b_{1}\right) \stackrel{i \gamma}{\longrightarrow}\left(i-1,0,1, b_{2}, b_{1}\right)$;

- $\quad\left(i, 1, s, 1, b_{2}, b_{1}\right) \stackrel{i \gamma}{\longrightarrow}\left(i-1,1, s, 1, b_{2}, b_{1}\right)$, if $b_{1} \neq K$.

- $\left(i, 1, s, 1, b_{2}, b_{1}\right) \stackrel{i \gamma+\theta}{\longrightarrow}\left(i-1,1, s, 1, b_{2}, b_{1}\right)$, if $b_{1}=K$ or $b_{2}+b_{1}=K$;

- $\quad\left(i, 1, s, 0, b_{2}, b_{1}\right) \underset{\theta}{\stackrel{\theta}{\longrightarrow}}\left(i-1,1, s, 0, b_{2}, b_{1}+1\right)$

- $\quad\left(i, 1, s, 0, b_{2}, b_{1}\right) \stackrel{\theta}{\longrightarrow}\left(i-1,1, s, 0, b_{2}, b_{1}\right)$, if $b_{1}=K$ or $b_{2}+b_{1}=K$;

- $\left(i, 0, w, b_{2}, b_{1}\right) \stackrel{i \sigma}{\longrightarrow}\left(i-1,1,1, w, b_{2}, b_{1}\right)$, for $w=0,1$;

- $\quad\left(i, 1, s, w, b_{2}, b_{1}\right) \stackrel{i \sigma(1-\delta)}{\longrightarrow}$ $\left(i-1,1, s, w, b_{2}, b_{1}\right)$, for $w=0,1$;

- $\quad\left(i, 1,1,0, b_{2}, b_{1}\right) \stackrel{\mu_{1}}{\longrightarrow}\left(i-1,0,1,0, b_{2}, b_{1}\right)$;

- $\left(i, 1, s, 0, b_{2}, b_{1}\right) \stackrel{\mu_{\mathrm{s}}}{\longrightarrow}\left(i-1,0, s, 0, b_{2}, b_{1}\right)$, if $\mathrm{s}=2,3$ and $b_{2}=0$;

- $\quad\left(i, 1, s, 0, b_{2}, b_{1}\right) \stackrel{\mu_{\mathrm{s}}}{\longrightarrow}\left(i-1,0,3,0, b_{2}, b_{1}\right)$, if $\mathrm{s}=2,3$ and $b_{2} \neq 0$;

- $\quad\left(i, 1, s, 1, b_{2}, b_{1}\right) \stackrel{\mu_{\mathrm{s}}}{\longrightarrow}\left(i-1,0,2,0, b_{2}, b_{1}\right)$, if $s=1,2,3$.

\section{Matrix $A_{1 i}$ has the following transitions:}

Entries of $A_{1 i}$ are transitions within level $i$. Let $b=0,1 ; w=0,1 ; b_{2}, b_{1}=1,2,3, \ldots K ; b_{2}+b_{1} \leq K$;

- $\left(i, 0, w, b_{2}, b_{1}\right) \stackrel{-\lambda-i \gamma-i \sigma-b_{1} \eta}{\longrightarrow}\left(i, 0, w, b_{2}, b_{1}\right)$, if $b_{1}=K$ or $b_{1}+b_{2}=K$ or $b_{1}+b_{2}<K$;

- $\left(i, 0, w, b_{2}, b_{1}\right) \stackrel{-\lambda-i \gamma-i \sigma}{\longrightarrow}\left(i, 0, w, b_{2}, b_{1}\right)$, otherwise . $\cdot\left(i, 1, s, w, b_{2}, b_{1}\right) \stackrel{\Delta}{\longrightarrow}\left(i, 1, s, w, b_{2}, b_{1}\right) \quad$ if $b_{1}=K$ or $b_{1}+b_{2}=K$ or $b_{1}+b_{2}<K$; where $\Delta=-\lambda-\mu_{\mathrm{s}}-i \gamma-i \sigma(1-\delta)-\theta-b_{1} \eta$.

$\cdot\left(i, 1, s, w, b_{2}, b_{1} \stackrel{\nabla}{\rightarrow}\left(i, 1, s, w, b_{2}, b_{1}\right)\right.$,otherwise; where $\nabla=-\lambda-\mu_{\mathrm{s}}-i \gamma-i \sigma(1-\delta)-\theta$

\section{STEADY STATE ANALYSIS}

The Model described here is a level dependent Quasi Birth Death (QBD) process. So, for further analysis 


\section{Available online at www.ijrat.org}

we use an algorithmic solution based on Neuts-Rao [13] truncation method. When we apply this method our process $\chi$ transforms to $\bar{\chi}$ with the infinitesimal generator :

$$
\bar{Q}=\left[\begin{array}{lllllllll}
A_{10} & A_{0} & \cdot & \cdot & \cdot & \cdot & \cdot & \cdot & \cdot \\
A_{21} & A_{11} & A_{0} & \cdot & \cdot & \cdot & \cdot & \cdot & \cdot \\
\cdot & A_{22} & A_{12} & A_{0} & \cdot & \cdot & \cdot & \cdot & \cdot \\
& & \ddots & \ddots & \ddots & & & \cdot & \cdot \\
\cdot & \cdot & \cdot & A_{2 N-1} & A_{1 N-1} & A_{0} & \cdot & . & \cdot \\
\cdot & \cdot & \cdot & \cdot & A_{2} & A_{1} & A_{0} & \cdot & \cdot \\
\cdot & \cdot & \cdot & \cdot & \cdot & A_{2} & A_{1} & A_{0} & \cdot \\
. & \cdot & \cdot & . & \cdot & \cdot & \ddots & \ddots & \ddots
\end{array}\right]
$$

where $\dot{A}_{1}=\dot{A}_{1 N}$ and $\dot{A_{2}}=A_{2 N}$

Let the steady state probability vector of the Markov process be $x=\left(x_{0}, x_{1}, x_{2}, \ldots\right)$. We take

$x_{N+i}=x_{N-1} R_{N}^{i+1}, \quad i=0,1,2, \ldots$

where $R_{N}$ is the minimal solution of the matrix quadratic equation $R_{N}^{2} A_{2 N}+R_{N} A_{1 N}+A_{0}=0$.

\section{STEADY STATE VECTOR}

Again, $x \bar{Q}=0$ leads to

and

$$
x_{N-i}=x_{N-i-1} R_{N-i} ; i=1,2, \ldots N-2
$$

$$
x_{1}=x_{0} R_{1} ; i=1,2, \ldots N-2
$$

where $R_{N-i}=-A_{0}\left(A_{1 N-i}+R_{N-i+1} A_{2 N-i+1}\right)^{-1}$ and $R_{1}=-A_{0}\left(A_{11}+R_{2} A_{22}\right)^{-1}$. Finally from $x_{0} A_{10}+$ $x_{1} A_{21}=0$ we find $x_{0}$ as the steady state distribution of finite state Markov chain with generator $A_{10}+$ $R_{1} A_{21}$. From (1), (2), (3), we get $x_{i}$ for $i=1,2,3, \ldots$. Now $x$ is calculated by dividing each $x_{i}$ with the normalizing constant $\sum_{i=0}^{\infty} x_{i} e$.

\section{SYSTEM STABILITY}

Theorem: The system under discussion is stable.

Proof: Consider the Lyapunov test function defined by $\phi(s)=i$ where $s$ is a state in level $i$. For a state in level $i$, the mean drift $y_{s}$ is given by

$$
\begin{aligned}
y_{s}= & \sum_{p \neq s}[\phi(p)-\phi(s)] q_{s p} \\
= & \sum_{s^{\prime}}\left[\phi\left(s^{\prime}\right)-\phi(s)\right] q_{s s^{\prime}}+\sum_{s^{\prime \prime}}\left[\phi\left(s^{\prime \prime}\right)-\phi(s)\right] q_{s s^{\prime \prime}}+ \\
& \sum_{s^{\prime \prime \prime}}\left[\phi\left(s^{\prime \prime \prime}\right)-\phi(s)\right] q_{s s^{\prime \prime \prime}}
\end{aligned}
$$

where $s^{\prime}, s^{\prime \prime}, s^{\prime \prime \prime}$ vary over states belonging to levels $i-1, i$ and $i+1$ respectively.

Then $\phi(s)=i, \phi\left(s^{\prime}\right)=i-1, \phi\left(s^{\prime \prime}\right)=i, \phi\left(s^{\prime \prime \prime}\right)=$ $i+1$. Now , $y_{s}=-\sum_{s^{\prime}} q_{s s^{\prime}}+\sum_{s^{\prime \prime \prime}} q_{s s^{\prime \prime \prime}}$.

Since $(1-\delta)>0$, for any $\epsilon>0$, we can find $K^{*}$ large enough such that $y_{s}<-\epsilon$ for any $s$ belonging to level $i \geq K^{*}$. Hence the theorem follows from Tweedie's [17] result.

\section{PERFORMANCE MEASURES}

Let $\xi=\left(\xi_{0}, \xi_{1}, \xi_{2}, \cdots\right)$ be our steady-state probability vector of the Markov process $\chi$. For the evaluation of system performance measures, we partition each $\xi_{i}, i \geq 0$ as follows: Let $\xi_{i}=\left(w_{i}, x_{i}, y_{i}, z_{i}\right)$ where each vector corresponds to the probability that the server is functioning with $i$ customers in the orbit.

- The probability that the server is idle, pidle $=\sum_{i=0}^{\infty} w_{i} e$
- The probability that the server is busy with an ordinary customer, $p$ sbor $=\sum_{i=0}^{\infty} x_{i} e$

- The probability that the server is busy with priority generated customer, $p s b p r=$ $\sum_{i=0}^{\infty} y_{i} e$

- The probability that the server is busy with interruption completed customers in $B_{2}$, $p s b b 2=\sum_{i=0}^{\infty} z_{i} e$

- The probability that the server is idle with customers in the orbit, psidco $=$ $\sum_{i=0}^{\infty} w_{i} e-w_{0} e$

- Expected number of customers in the orbit, exor $=\sum_{i=1}^{\infty} i \xi_{i} e$

- Expected number of customers in the orbit when the server is idle, exsidle $=\sum_{i=1}^{\infty} i w_{i} e$

- Expected number of customers in the orbit when the server is busy with an ordinary customer, exsbor $=\sum_{i=1}^{\infty} i x_{i} e$

- Expected number of customers in the orbit when the server is busy with priority generated customer, exsbpr $=\sum_{i=1}^{\infty} i y_{i} e$

- Expected no. of customers in the orbit when the server is busy with interruption completed customer, exsbb2 $=\sum_{i=1}^{\infty} i z_{i} e$

- Successful retrial rate, surerate $=$ $\sigma \sum_{i=1}^{\infty} i w_{i} e$

- Overall retrial rate, ovrerate $=\sigma \sum_{i=1}^{\infty} i \xi_{i} e$

- The fraction of success rate of retrial, frsurerate $=\frac{\text { surerate }}{\text { ovrerate }}=\frac{\sum_{i=1}^{\infty} i w_{i} e}{\sum_{i=1}^{\infty} i \xi_{i} e}$

Let $\xi_{i}=\zeta_{i}\left(b, s, w, b_{2}, b_{1}\right)$ where $\zeta_{i}\left(b, s, w, b_{2}, b_{1}\right)$ is a row vector corresponding to $N_{2}(t)=b, S(t)=s$, $N_{3}(t)=w, N_{4}(t)=b_{2}, N_{5}(t)=b_{1}$ with $b=0,1 ; s=1,2,3 ; w=0,1 ; b_{2}, b_{1}=$ $0, \cdots, K ; b_{2}+b_{1} \leq K$.

- Expected number of priority generated customers lost from the system, exprl $=\sum_{i=1}^{\infty} \sum_{b=0}^{1} \sum_{s=1}^{3} \sum_{b_{2}=0}^{K} \sum_{b_{1}=0}^{K-b_{2}} i \zeta_{i}\left(b, s, 1, b_{2}, b_{1}\right) e$

- Expected number of interrupted customers lost from the system.

exinl $=\sum_{i=1}^{\infty} \sum_{b=0}^{1} \sum_{s=1}^{3} \sum_{b_{2}=0}^{K} i \zeta_{i}\left(b, s, 1, b_{2}, K-b_{2}\right) e$ 


\section{Available online at www.ijrat.org}

\section{NUMERICAL ILLUSTRATION}

(a) Effect of changing $\gamma$ in some performance measures :

Let $\sigma=0.6, \eta=3.6, \delta=0.3, \theta=2.4, \lambda=2.2, \mu_{1}=$ $0.4, \mu_{2}=1.9, \mu_{3}=0.3$ and compute the following measures using different $\gamma$ values and plot Figure 1 and Figure 2. From Figure 1 we observe that when $\gamma$ increases the expected number of customers in the orbit when the server is busy with priority generated customers decreases. From Figure 2 we observe that when $\gamma$ increases successful retrial rate decreases gradually and overall retrial rate also decreases. But the fraction of success rate of retrial increases with the increase of $\gamma$.

(b) Effect of changing $\theta$ in some performance measures :

Let $K=1, \sigma=0.6, \eta=1.6, \gamma=5, \delta=0.3, \lambda=$ $5.2, \mu_{1}=1.5, \mu_{2}=2.5, \mu_{3}=1.0$ and compute the following measures using different $\theta$ values and plot Figure 3. From Figure 3 we observe that when $\theta$ increases the expected number of customers in the orbit when the server is busy with interruption completed customers increases initially and reaches a maximum and then decreases.

(c) Effect of changing $\theta$ in some performance measures :

Let $\quad \sigma=0.6, \eta=1.6, \gamma=5, \delta=0.3, \lambda=5.2$, $\mu_{1}=1.5, \mu_{2}=2.5, \mu_{3}=1.0$ and compute the expected number of interrupted customer lost using different $\theta$ values and for $\mathrm{K}=1,2$ plot Figure 4 .

From Figure 4 we observe that when $\theta$ increases the expected number of interrupted customers lost increases initially and reaches a maximum and then decreases. Also when buffer size $\mathrm{K}$ increases, the expected number of interrupted customers lost decreases.

(d) Effect of changing $\gamma$ and $\lambda$ in some performance measures :

Let $\mathrm{K}=2, \sigma=0.6, \eta=1.6, \theta=2.4, \delta=0.3, \mu_{1}=$ $1.5, \mu_{2}=4.0, \mu_{3}=1.0$ and compute the expected number of priority generated customers lost using different $\gamma$ values and plot Figure 5. From Figure 5 we observe that when $\lambda$ increases the expected number of priority generated customers decreases gradually and when $\gamma$ increases, the expected number of priority generated customers lost increase very fast for smaller values of $\lambda$.
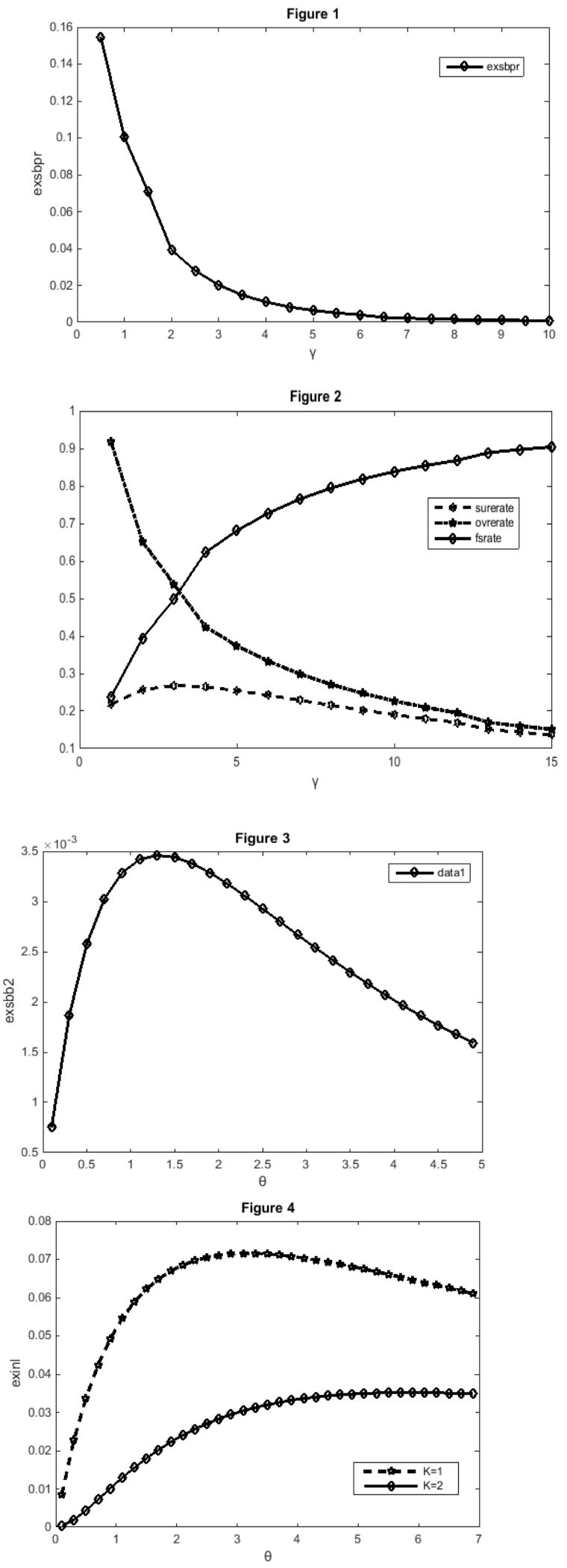


\section{Available online at www.ijrat.org}

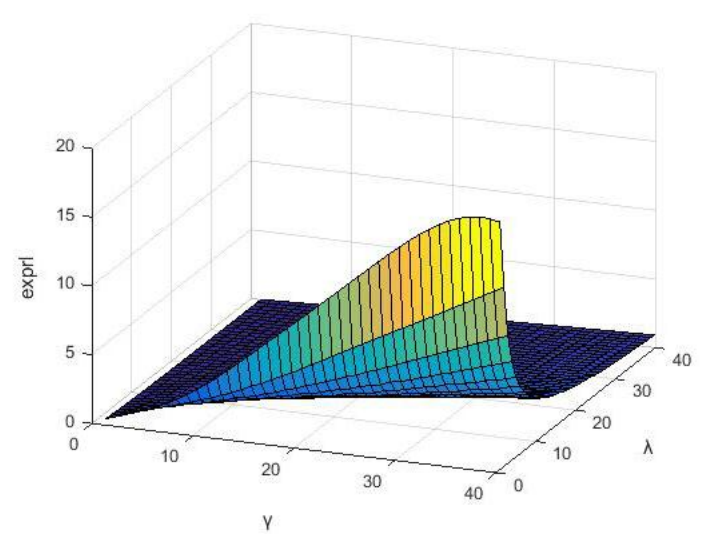

\section{CONCLUSION}

A single server retrial queueing system with selfgeneration of priorities is analyzed in this paper. The arrival of customers are according to Poisson process and service times follow different Exponential distributions. The interruption we discussed here is customer induced interruption. Performance measures required for an appropriate system designing were computed and numerically analyzed.

\section{Acknowledgment}

The authors are thankful to Professor A. Krishnamoorthy for constructive suggestion and advice in the entire work of this paper. Support from the University Grants Commission (sanction no.FIP/12th Plan/KLKE029 TF-36) is gratefully acknowledged.

\section{REFERENCES}

[1] Gennadi Falin and James GC, Templeton. Retrial queues, voiume 75, CRC Press, 1997.

[2] Gennadi Falin. A survey on retrial queues, Queueing Systems, 7(2): 127-167,1990.

[3] Brahimi, M, and Worthington, DJ, Queueing models for out-patient appointment systems $-A$ case study. Journal of the Operational Research Society, 42(9):733-746,1991.

[4] A Gomez-Corral. Analysis of a single-server retrial queue with quasi-random input and nonpreemptive priority. Computers \& Mathematics with Applications, 43(6-7):767-782,2002.

[5] A Gomez-Corral. A Krishnamoorthy and Viswanath C Narayanan. The impact of selfgeneration of priorities on multi-server queues with finite capacity. Stochastic Models,21(23):427-447,2005.

[6] Varghese Jacob, Srinivas R Chakravarthy, and Achyutha Krishnamoorthy. On customer-induced interruption in a service system. Stochastic Analysis and Applications, 30(6):949-962,2012.

[7] Narendra Kumar Jaiswal. Priority queues. Elsevier, 1968.
[8] A. Krishnamoorthy, S Babu, and Viswanath C. Narayanan. MAP/(PH, PH)/c queue with selfgeneration of priorities and non-preemptive service. Stochastic Analysis and Applications, 26(6):1250-1266, 2008.

[9] A. Krishnamoorthy, S Babu, and Viswanath C. Narayanan. MAP/(PH, PH)/1 queue with selfgeneration of priorities and non-preemptive service. European Journal of Operational Research, 195(1):174-185, 2009.

[10] Achyutha Krishnamoorthy, Padinhare K Pramod, and Srinivas R Chakravarthy. Queues with interruptions: a survey. Top, 22(1):290-320, Springer, 2014.

[11] Viswanath C. Narayanan and A. Krishnamoorthy. Queues with retrial/self-generation of priorities/postponement of work and some related reliability problems. Ph.D. Thesis, 2005.

[12] Marcel F Neuts. Matrix-geometric solutions in stochastic models: an algorithmic approach. Courier Corporation, 1981.

[13] Marcel F Neuts and BM Rao. Numerical investigation of a multiserver retrial model. Queueing systems, 7(2):169-189, 1990.

[14] John F Shortle, James M Thompson, Donald Gross, and Carl M Harris. Fundamentals of queueing theory. John Wiley \& Sons, 2018.

[15] H Takagi. Queueing analysis: Vacations and priority system, vol. i. INorth-Holland, Amsterdam, 1991.

[16] IDS Taylor and JGC Templeton. Waiting time in a multi-server cut off-priority queue, and its application to an urban ambulance service. Operations Research, 28(5):1168\{1188,1980.

[17] Richard L Tweedie. Sufficient conditions for regularity, recurrence, and ergodicity of Markov processes. In Mathematical Proceedings of the Cambridge Philosophical Society, volume 78, pages 125-136. Cambridge University Press, 1975.

[18] Tao Yang and James G. C. Templeton. A survey on retrial queues. Queueing systems, 2(3): 201233, 1987. 